\title{
The management of subacute and chronic vascular aphasia: an updated review
}

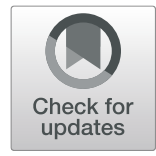

Samwel Sylvester Msigwa ${ }^{1,2^{*}}$ (D) and Xianglin Cheng ${ }^{1,3}$

\begin{abstract}
Background: Post-stroke aphasia (PSA) is an impairment of the generation or comprehension of language due to acute cerebrovascular lesions. Subacute phase span the 7th day to 24 weeks post-onset while $>6$ months is termed chronic phase. Language recovery does not arise immediately in chronic PSA, unlike the acute phase. The majority of the treatment modalities in these two PSA phases are still in the infancy stage, facing dilemmas and considered experimental requiring constant updates. Hence, we aimed to upgrade the existing literature regarding available PSA management options, advances, and drawbacks pertaining to subacute and chronic phases.

Main text: In this review, we analyzed the management options for subacute and chronic vascular aphasia. MEDL INE, through PubMed, ScienceDirect, and Google Scholar were explored for English studies by utilizing the terms "stroke aphasia" Plus "vascular aphasia"; 160,753 articles were retrieved. The latest studies, published from 2016 to July 2020, were selected. Article headings and abstracts were analyzed for relevance and filtered; eventually, 92 articles were included in this review. Various management options were extracted as follows: noninvasive brain stimulation (NIBS), technology-based therapies, speech-language therapy (SLT), pharmacotherapy, music-based therapies, and psychosocial interventions.

Conclusion: The PSA therapy evolves towards more intense SLT therapy, yet the optimal dosage of the emerging high-intensity therapies is controversial. As spinal and cerebellar NIBS, Telespeech, and E-mental health mark PSA's future, distinct pharmacological options remain a dilemma. Across the continuum of care, PSA-depression comorbidity and inadequate PSA post-discharge education to patient's families are the significant therapeutic challenges. Future therapeutic mechanisms, optimal dose/timing, and tolerability/safety exploration are obliged.
\end{abstract}

Keywords: Language, Aphasia, Depression, Speech therapy

\section{Background}

Over the last two decades, advancement in neural cognition sciences has pointed out that the language role was not restricted to the classical language domains and pointed favored cerebral regions, which beforehand was not confirmed [1]. Post-stroke cases are frequently harmed by disabilities that deter quality of life; yet, there are numerous options for rehabilitation choices and

\footnotetext{
* Correspondence: neurovascular7@gmail.com

'Department of Neurology, The Clinical Medicine School of Yangtze

University, The First Affiliated Hospital of Yangtze University, Jingzhou 424023, Hubei, China

${ }^{2}$ Mirembe National Mental Health Hospital, Dodoma, Tanzania

Full list of author information is available at the end of the article
}

approaches. Hence, it is vital to locate the most efficient modality [2].

Aphasia refers to an impairment of verbal communication, comprehension or speech production, and the ability to read or write secondary to brain injury. Poststroke aphasia (PSA) is the most common form of aphasia, mostly due to left hemispheric lesions [3-5]. Based on the Stroke Recovery and Rehabilitation Roundtable proposed framework definitions for critical timepoints post-stroke, the subacute post-stroke phase spans the 7 th day to the 6th month post-onset. Subclassification involves early subacute (7th to 90th day) and late 
subacute (90th to 180th day). In contrast, beyond 180 days (6 months) is termed the chronic phase [6].

Classic aphasic syndromes correlate with the particular vascular territory. Grouping post-stroke victims by affected vascular territory aids in the localization of particular aphasia phenomenon to the related vessel affected. The advent of imaging technologies yields the opportunity to prove that conventional aphasic syndrome corresponds with particular vascular regions. Clinically, Wernicke's aphasia localizes lower-division middle cerebral artery strokes; Broca's aphasia localizes superior division, while the proximal portion occlusion is predicted better by global aphasia [7]. Therefore, PSA (vascular aphasia) is the damage or impairment of the generation or comprehension of language either in the form of speaking or writing resulting from a cerebrovascular event emerging from ischemia or hemorrhagic insult [6-8].

A series of researches confirmed treatment-induced language advances clinically and neurophysiologically. Unlike in the acute phase, in the chronic PSA stage, language repairs regularly do not arise immediately; therefore, considering natural restoration means is necessary. Chronic PSA cases are independent of neural plasticities, such as reduced cerebral edema. Hence, the post-stroke restructuring of language is highly scrutinized in victims with chronic vascular aphasia (CVA) [9].

The contemporary data links the functional reconstruction of language in CVA with neuroplastic alterations in both cerebral hemispheres, more marked at the left hemispheric (LH) augmentations throughout the early staging of language recovery [10].

Previous studies documented that the most significant changes in the neural structure for language happen in the initial regeneration steps. Contemporary post-stroke motor recovery studies revealed an enhanced sensitivity to treatment even beyond the critical window (3 to 6 months) post-onset. Similarly, the majority of language neuroplasticity proved to occur in the subacute phase, followed by residual recovery from compensatory mechanisms throughout the chronic phase $[11,12]$. There are several PSA recovery prognostic factors, the baseline severity being the single most significant PSA outcome predictor at 3 months and other factors being subcortical white matter tract integrity and preserved left hemispheric cortical regions' structural integrity [11-13].

Recovery in PSA exhibits a non-linear pattern, due to differences in recovery mechanisms and patterns associated with stroke duration. Mechanisms in the subacute phase are characterized mainly by cerebral edema resolution, and several pathological processes return to the baseline state. Additional potential mechanisms in this phase include synaptogenesis and axonal-dendritic regeneration. Further, perilesional cortical neurogenesis are reported. Recent studies on Wernicke's aphasia offer proof that a rapid auditory temporal processing may offer comprehension outcomes in the subsequent (chronic) phase [14, 15].

Abnormal perfusion continues into the chronic phase, even post-ischemic penumbra resolution. Despite the subsiding physiological changes, the mechanisms that facilitate plasticity mainly acquired due to environmental experience remain intact. Further, an advent of fMRI studies associate chronic PSA recovery mechanism with the activation in the dominant hemisphere (left). The atypical task-evoked responses during semantic processing are associated with damage to the posterior temporoparietal areas-additionally, the suboptimal CVA recovery is linked to disruption network integrity and white matter connectivity $[15,16]$.

In PSA cases, the purpose of clinical rehabilitation is to regain failed functions and prevent the acquired pathologies by administering training. Lately, conventional clinical practices began to experiment new proposals from neurophysiological studies in primates. Consequently, the therapy should incorporate mastery of both behavioral aspects and neurophysiological perspectives [17].

Recently, in a wave of attention in rehabilitation, psychiatric and neurological utilization of noninvasive brain stimulation (NIBS) approaches that are mainly repetitive transcranial magnetic stimulation (rTMS) and transcranial direct current stimulation (tDCS) have been reported [18]. Despite NIBS convincing post-stroke language restoration, the mechanisms, optimal stimulated cerebral regions for linguistic improvement, stimulation frequencies, and the most desirable electrode positions for PSA cases prevail a point of debate [1922].

Similarly, a long-standing discussion has encompassed a suitable amount of CVA speech-language therapy (SLT). Nevertheless, the efficient performance of intensive modalities is yet to be entirely appreciated [23]. Even though speech-language pathologists (SLPs) learn about PSA cases' communicative necessities contrasted to other affiliates in stroke rehabilitation, their counseling practice still reported facing shallow understanding, ability, and courage. Therefore, modernizing information and reexamining what coaching empowers SLPs to consider competent, self-confident, and skillful is mandatory [24].

The proof supporting self-management in stroke is proliferating, yet, there is inadequate evidence to demonstrate PSA self-management efficacy [25]. Furthermore, practice in SLT for evaluation is novel; hence, its effectiveness and potential for rehabilitation in PSA prevail unexplored [26]. On the other hand, around $60 \%$ of 
CVA cases documented co-occurring with depression; similarly, online-based mental health practice was reported to be increasingly employed in the management of post-stroke depression (PSD) [27].

Owing to these remarkable advancements and knowledge gaps, in this review, we analyzed the management of options for subacute and chronic vascular aphasia. ScienceDirect, Google Scholar, and MEDLINE, through PubMed, were explored for English studies by utilizing the terms "stroke aphasia" Plus "vascular aphasia"; a total of 160,753 articles were retrieved. The latest studies, published from 2016 to July 2020, were selected. Article headings and abstracts were analyzed for relevance and filtered, and eventually, 92 articles were incorporated.

\section{The management of subacute and chronic vascular aphasia}

Noninvasive neuromodulation technologies

Neuromodulation technologies (Table 1) also known as NIBS, is encouraging means for neurorehabilitation in
PSA $[18,28]$. The most recent meta-analyses authenticated NIBS as an efficient therapy in promoting the recovery when coupled with SLT compared with monotherapy. rTMS are exhibiting an advantage of effectiveness for both subacute vascular aphasia (SVA) and CVA, bearing more safety but are more costly compared with tDCS. On the contrary, tDCS is user-friendly, offering quick utilization at home, with little expense but limited practicability, and satisfactory only for CVA [2931].

Brain stimulation techniques set a novel PSA approach; Spielmann and colleagues strengthened the probability of determining an optimal electrode position, hence registering the necessity for the prospective studies to analyze the usefulness of the approved electrode arrangement [21].

NIBS can affect mechanisms of synaptic plasticity, targeting distinct sections across the cortical and subcortical regions. Compared with natural mechanisms, NIBS stimulation is considerably complicated, emerging from

Table 1 Summary of studies on subacute and chronic vascular aphasia (January 2016-February 2020) evaluating the potency of noninvasive neuromodulation technologies

\begin{tabular}{|c|c|c|c|c|c|}
\hline Author & $\begin{array}{l}\text { Case } \\
(\boldsymbol{n})\end{array}$ & Intervention & $\begin{array}{l}\text { Post- } \\
\text { stroke } \\
\text { onset }\end{array}$ & Therapeutic duration/intensity & Outcome \\
\hline $\begin{array}{l}\text { Meinzer } \\
\text { et al.,2016 }\end{array}$ & 26 & $\begin{array}{l}\text { Intensive } \\
\text { naming + } \\
\text { tDCS }\end{array}$ & $\begin{array}{l}>12 \\
\text { months }\end{array}$ & $\begin{array}{l}\text { Naming therapy }(14 \text { days } / 1.5 \mathrm{~h} / \text { day }) \times \\
2 \text { per day prior stimulation }\end{array}$ & $\begin{array}{l}\text { Multi-session tDCS provoke long-lasting enhancement } \\
\text { of therapeutic outcome }\end{array}$ \\
\hline $\begin{array}{l}\text { Carlson et al. } \\
2016\end{array}$ & 1 & rTMS + SLT & $\begin{array}{l}30 \\
\text { months }\end{array}$ & 2 weeks/ rTMS + SLT & rTMS is safe in pediatric PSA \\
\hline $\begin{array}{l}\text { Sebastian } \\
\text { et al. } 2017\end{array}$ & 1 & $\begin{array}{l}\text { Cerebellar } \\
\text { tDCS + BT }\end{array}$ & 5 years & $\begin{array}{l}15 \text { sessions / } \\
60 \text { days apart. }\end{array}$ & $\begin{array}{l}\text { Anodal cerebellar tDCS + BT is more efficient than BT } \\
\text { alone }\end{array}$ \\
\hline $\begin{array}{l}\text { Marangolo } \\
\text { et al. } 2017\end{array}$ & 14 & tsDCS & $\begin{array}{l}\geq 6 \\
\text { months }\end{array}$ & 3 weeks / $20 \mathrm{~min} / 2 \mathrm{~mA}$ & tsDCS promotes verb recovery in chronic PSA \\
\hline $\begin{array}{l}\text { Harvey et al. } \\
2017\end{array}$ & 09 & rTMS & $\begin{array}{l}55 \\
\text { months }\end{array}$ & 10 days/600 pulses & $\begin{array}{l}\text { rTMS offers long-lasting enhancements in picture } \\
\text { naming }\end{array}$ \\
\hline $\begin{array}{l}\text { Woodhead } \\
\text { et al. } 2018\end{array}$ & 21 & $\begin{array}{l}\text { iReadMore VS } \\
\text { tDCS }\end{array}$ & $>1$ year & $\begin{array}{l}4 \text { weeks/ } 34 \mathrm{~h} \text { iReadMore }+11 \\
\text { stimulation sessions }\end{array}$ & $\begin{array}{l}\text { iReadMore confirmed in advancing the reading } \\
\text { proficiency for trained words in central alexia }\end{array}$ \\
\hline $\begin{array}{l}\text { Spielmann } \\
\text { et al. } 2018\end{array}$ & 13 & $\begin{array}{l}\text { tDCS + word- } \\
\text { finding }\end{array}$ & $\begin{array}{l}\geq 6 \\
\text { months }\end{array}$ & 3 sessions, 3 days apart / 1 month & $\begin{array}{l}\text { There is probability of determining an optimal } \\
\text { electrode position }\end{array}$ \\
\hline $\begin{array}{l}\text { Hu et al. } \\
2018\end{array}$ & 40 & $\begin{array}{l}\text { LF-rTMS } \\
\text { VS HF-rTMS }\end{array}$ & $\begin{array}{l}>1 \\
\text { month }\end{array}$ & $\begin{array}{l}\text { HF-rTMS }(10 \mathrm{~Hz}) \\
\text { LF-rTMS }(1 \mathrm{~Hz})\end{array}$ & $\begin{array}{l}\text { Low-frequency rTMS is more efficient than the high } \\
\text { frequency. }\end{array}$ \\
\hline $\begin{array}{l}\text { Szaflarski } \\
\text { et al. } 2018\end{array}$ & 12 & iTBS + mClAT & & $\begin{array}{l}\mathrm{mCIAT} 45 \mathrm{~min} / 10 \text { days } \\
1 \text { session iTBS/10 days } \\
\text { Before mCIAT }\end{array}$ & iTBS + mCIAT achievable and safe \\
\hline $\begin{array}{l}\text { Vila-Nova } \\
\text { et al. } 2019\end{array}$ & 13 & $\begin{array}{l}\text { tDCS + word- } \\
\text { finding }\end{array}$ & $\begin{array}{l}15-70 \\
\text { months }\end{array}$ & $\begin{array}{l}-6 \text { months SLT } \times 1 \text { for week } \\
-3 \text { weeks tDCS }\end{array}$ & tDCS effectivity in articulation but not word count \\
\hline $\begin{array}{l}\text { Harvey et al. } \\
2019\end{array}$ & 11 & CTBS & $\begin{array}{l}\geq 6 \\
\text { months }\end{array}$ & 600 pulses & $\begin{array}{l}\text { CTBS offer long-lasting enhancements in picture } \\
\text { naming }\end{array}$ \\
\hline $\begin{array}{l}\text { Heikkinen } \\
\text { et al. } 2019\end{array}$ & 17 & rTMS + ILAT & $\begin{array}{l}\geq 12 \\
\text { months }\end{array}$ & 2 weeks & $\begin{array}{l}\text {-ILAT exhibited effectiveness for the chronic PSA phase } \\
\text {-rTMS did not yield notable results }\end{array}$ \\
\hline $\begin{array}{l}\text { Georgiou } \\
\text { et al. } 2019\end{array}$ & 02 & cTBS & $\begin{array}{l}20-25 \\
\text { months }\end{array}$ & 600 pulses & $\begin{array}{l}\text { CTBS as monotherapy improve promote language } \\
\text { recovery }\end{array}$ \\
\hline
\end{tabular}

mCIAT Modified constraint-induced aphasia therapy, tsDCS Transcutaneous spinal direct current stimulation, $r T M S$ repetitive transcranial magnetic stimulation, $H F-$ rTMS high-frequency rTMS, LF-rTMS low-frequency rTMS, CTBS continuous theta burst stimulation, ILAT intensive language-action therapy, iTBS intermittent theta burst suppression, BT behavioral therapy, SLT speech-language therapy 
excitatory and inhibitory outcomes. NIBS protocols lead to persistent impacts on cortical excitability. This observation reveals the synaptic mechanisms of long-term potentiation (LTP) or depression (LTD). Nevertheless, recent studies indicate that LTP/LTD aspects alone are inadequate to justify the initial and long-term modifications observed following short-term NIBS experiences $[11,32]$.

Mechanism of tDCS focuses on transmitting a little polarizing electrical current, (1-2 mA), modulating cortical activity by stimulating neuronal resting membrane potentials, which enhance cognition and conduct. Anodal tDCS renders neuron depolarization, boosting cortical excitability, while cathodal tDCS acts by neuronal hyperpolarization, reducing excitability. tDCS, coupled with behavioral sessions, have been correlated with enduring changes in both performed and neuronal activities $[1,18,32,33]$.

Previous researches displayed transcutaneous spinal direct current stimulation (tsDCS) capabilities in modulating spinal cord. In these earlier studies, tsDCS mechanisms directed on the lemniscal and nociceptive spinal arrangements. Marangolo and colleagues, in the most contemporary study, shed light, highlighting the spinal cord as a bridge for conveying tsDCS effected changes into cerebral networks to promote verb processing. The hypothesized mechanism links anodal tsDCS with the inhibition of the ascending tonic pathways to the cortex. This theory displays tsDCS, decreasing the activity into the sensorimotor areas. Paradoxical neuronal potentiation of their role is the outcome. Further, inhibitory current is thought to decrease cortical inhibitory interneuron excitability. The last speculated concept concentrated on anodal tsDCS effect on glutamate and GABA (decreased levels) into the sensorimotor cortices pointing to an enhanced function [1].

Identify the best region for the tDCS placement region has been in a milestone. Previous studies authenticated proof that placement of anodal tDCS over peri-lesional LH regions or cathodal tDCS over the right hemisphere (RH) regions can augment language outcomes. Likewise, several studies have shown the benefit of utilizing both electrodes (RH cathodal tDCS /LH anodal tDCS). On the contrary, the most contemporary study applied right cerebellar neuromodulation to increase spelling restoration in a CVA case. Extended span (up to 5/week) and intensity (15 sessions) thought to play a role in the notable spelling skills improvement observed. Further, the role neuronal LTP in synaptic connectivity and cerebellum facilitation by tDCS in skill learning is another possible mechanism. However, this study was a case-based research on a single participant, warranting future extensive studies [19].
In one randomized controlled trial (RCT) employing 26 CVA cases post highly intensive naming treatment plus tDCS, proved for the first moment that multisession tDCS could provoke long-lasting enhancement of therapeutic result by applying M1 tDCS montage [34]. Another RCT tested the impact of left inferior frontal gyrus (IFG) anodal tDCS combined with the iReadMore app on reading skills for alexia-aphasia comorbidity. Based on 21 cases, iReadMore confirmed to advance the reading proficiency for trained words, with excellent long-term outcome [35]. An up-to-date metaanalysis on repetitive tDCS has demonstrated the probability of intensifying picture-naming correctness in PSA sufferers. Hence, uncovering the prospect of providing tDCS therapeutic to improve language rehabilitation in clinical rounds, yet, further investigations to validate these prefatory outcomes is needed [36].

TMS creates cerebral hyperpolarization/depolarization through weak currents. A coil was set on the scalp and a pulse transmitted to the skull. The application above Broca's area was linked to enhance language outcomes. Precisely, TMS centered on fluxing magnetic field production, which in turn produces a current in underlying cortical neurons, generating depolarization. As a result, TMS can be used to manipulate cortical capacity in a focal way [18].

In one systemic review by Dionísio and colleagues, in 2018, most included studies stated statistically meaningful functional enhancements, promoting the application of TMS for PSA rehabilitation [2]. Further, based on a PSA pediatric case report, SLT coupled with rTMS seems safe, as the post-therapy fMRI displayed enhanced left perilesional IFG activity and connectivity [37]. Similarly, inhibitory rTMS to regions of the right IFG of CVA patients proved to present long-lasting enhancements in picture naming production and cortical activation [20].

A recent proof authenticates that low-frequency rTMS (LF-rTMS) is more efficacious than the high-frequency rTMS; still, studies necessitated examining the neuronal mechanisms that hold the variations in functional restoration witnessed among the stimulation techniques [22]. Likewise, the most up-to-date reappraised guidelines on the curative effect of rTMS recommend that LF-rTMS be used for the right IFG in motor CVA, despite the guideline endorsement of rTMS advantages, but it is yet to attain clinical significance levels [38].

In a case report concerning a PSA female patient in her thirties, the application of rTMS coupled with SLT conferred intensified cerebral functioning [39]. On the contrary, one study based on 17 victims with chronic PSA in whom rTMS coupled with intensive languageaction therapy (ILAT) was exercised, rTMS did not yield notable results. Instead, ILAT exhibited effectiveness for 
the chronic PSA phase; the findings were reported from evidence-based PSA therapies [28]. In one explorative research with a limited number of 2 participants, continuous TBS at the right pars triangularis as a monotherapy proved the ability to promote language symptoms in the chronic PSA with no unfavorable outcomes during the therapeutic and follow-up duration [40].

Continuous theta-burst stimulation (cTBS) is an inhibitory form of rTMS to the right of the triangular part of the IFG used in CVA. It can intensify naming by aiding phonological access throughout word retrieval, forming a unique chance for PSA cases with naming impairment in the chronic platform [41]. iTBS is correlated with improved left lateralization of IFG activity during verb production, registering neuroplastic modifications associated with iTBS employed on residual lefthemisphere language regions in $\mathrm{CV} \mathrm{A}[42]$.

\section{Speech-language therapy}

In regular speech, illustrations of things and acts are customarily actualized as nouns and verbs. Gleichgerrcht and colleagues recently investigated separate neural arrangement assistance and found words for activities linked with a frontal circuitry required in motor command and programming. Comparatively, words for things are associated with posterior networks [43]. Moreover, Kjellén and colleagues demonstrated that PSA victims could verbalize their literacy encounters and beliefs, warning clinicians to compose or alter their literacy evaluations and intervention strategies accurately [44]. Further, PSA survivors recognized to offer added meaning-ladened gesticulations to counterbalance verbal production shortfalls. These observations were suggesting the beneficial impact of multimodal information provided by co-speech gesticulations [45]. Hence, new word training performance seems to offer acquisition in anomia recovery [46].

Unlike other PSA therapeutic modalities lacking full recommendations, contemporary rehabilitation guidelines propose SLT for PSA [19, 47]. One study established that, amid community-dwelling PSA victims, majority of SLT sessions implemented face to face compared with group therapy and telehealth, regularly executed by qualified SLPs in more than $70 \%$ of the cases and infrequently by SLP assistants, for 1-60 min per session in 2 weeks apart [48].

The significance of intensive, comprehensive aphasia plans (Table 2) has developed due to increasing demand for CVA treatments, now the proof to confirm language and life status change in PSA, yet, the retrospective character of the studies is a significant drawback [49].

Constraint-induced aphasia therapy (CIAT) is known to be an intensive, high-dose management directed at improving verbal output in a group setting of 2-3 subjects entirely centering on verbal rather than nonverbal activities. The intensified CIAT protocol, which incorporates written hints (CIAT-Plus) and multimodal aphasia treatment (M-MAT), will be concluded in the future large RCT (COMPARE) with 216 participants. Completion will permit more efficient prescriptions for PSA, and cost-effectiveness data will render support for marketing [50]. However, CIAT/ILAT has been determined to be highly useful in improving language shortfalls in CVA as well as in subacute vascular aphasia (SVA). In one study where ILAT was rendered for $10 \mathrm{~h}$ weekly for 4 weeks, cerebral activation improvement post-treatment throughout sentence processing was observed, authenticating that high-intensity language therapy promotes

Table 2 Summing researches published (2016-February 2020) appraising the effectiveness of speech-language therapy on subacute and chronic vascular aphasia

\begin{tabular}{|c|c|c|c|c|}
\hline Author & $\begin{array}{l}\text { Sample } \\
\text { size }(\boldsymbol{n})\end{array}$ & Intervention type & $\begin{array}{l}\text { Duration } \\
\text { post onset }\end{array}$ & Outcome \\
\hline $\begin{array}{l}\text { Dignam } \\
\text { et al. } 2016\end{array}$ & 30 & $\begin{array}{l}\text { SFA/PCA }+ \\
\text { computer therapy }\end{array}$ & $>4$ months & New word training correlated with therapeutic outcomes \\
\hline $\begin{array}{l}\text { Duncan } \\
\text { et al. } 2017\end{array}$ & 19 & IAT & $>5$ months & Intensive IAT correlates with therapeutic benefit (narrative production) \\
\hline $\begin{array}{l}\text { Kjellén et al. } \\
2017\end{array}$ & 12 & $\begin{array}{l}\text { Literacy } \\
\text { intervention }\end{array}$ & $1.5-25$ years & Literacy intervention facilitates verbalization literacy encounters and beliefs \\
\hline $\begin{array}{l}\text { Lucchese } \\
\text { et al. } 2018\end{array}$ & 14 & ILAT/CIAT & $>1$ year & High-intensity language therapy promotes language recovery in chronic PSA \\
\hline $\begin{array}{l}\text { Preisig et al. } \\
2018\end{array}$ & 20 & MCA & $\geq 6$ months & $\begin{array}{l}\text { PSA survivors recognized to offer added meaning-ladened gesticulations to counter- } \\
\text { balance verbal production shortfalls post therapy. }\end{array}$ \\
\hline $\begin{array}{l}\text { Kaviani et al. } \\
2018\end{array}$ & 2 & MCP & $3 / 8$ years & The MCP enhance the communication abilities in patients with chronic PSA \\
\hline $\begin{array}{l}\text { Stahl et al. } \\
2018\end{array}$ & 30 & ILAT & $\geq 1$ year & Increasing treatment intensity has no added outcome value. \\
\hline
\end{tabular}

IAT imitation-based aphasia therapy, ILAT intensive language-action therapy, CIAT constraint-induced aphasia therapy, MCP multimodal communication program, MCA multimodal communication in aphasia, SFA semantic features analysis, PCA phonological components analysis 
language recovery in chronic PSA over a short period [9].

Current data has fired the debate on the purpose of massed therapies in CVA. Early findings proposed a weekly dosage of moderate ILAT in a range of $5-10 \mathrm{~h}$ as adequate for ensuring enhanced language execution. Nevertheless, no definitive resolution, whether highlyintensive ILAT in a weekly dose over and above $10 \mathrm{~h}$, points to additional SLT profits. The primary RCT, by Stahl and colleagues, in 2018 which presented proof on various ranges of massed therapy by analyzing weekly 12-h and 6-h therapies proved lack of added therapeutic intensity benefit for an extra $2 \mathrm{~h}$ of daily therapy within a month $[23,47]$.

Imitation-based aphasia therapy (IAT) is a variety of action-observing therapy applying engagement of the given anatomical network bearing action observation and execution to improve function post-neurological injury. Based on 19 PSA cases in a chronic phase, 6 weeks of intensive IAT, the intensity of therapy was observed to correspond with therapeutic gain. In contrast, inadequate representation and compromised therapeutic compliance due to unexamined variables, such as attention, were marked as limitations [51].

In CIT, non-verbal actions are considered to intervene in therapy, and patients are consequently compelled to speech modality. Comparatively, multimodal therapeutics apply nonverbal modalities to cue word retrieval. Multiple nonverbal modalities may be coupled in therapy to potentiate the cueing of verbal output. Multimodality aphasia therapy offers a highly intensive multimodal approach that employs structured cueing ranks of writing, gesticulation, and drawing to cue word retrieval [52].

The conventional procedure (unimodal approach) centered on the therapist guiding a particular modality independently. A new augmentative alternative communication, multimodal communication program (MCP) approach, in CAV can enhance the communication abilities in CVA cases [53].

\section{Technology-based therapy}

High technology media designate a compensatory approach to intensify communicative abilities in PSA individuals; scientific conclusions from aphasia therapy are adequately represented by advanced study designs and outcomes reporting [54]. Now computerized SLT can be confirmed as a supportive approach to PSA treatment, operated at a low cost compared with conventional faceto-face SLT; coming computerized rehabilitation programs will conceivably improve the effectiveness of accredited applications in CVA [55].

In a study comprising 20 PSA participators offered with 12 computer-based therapy sessions for 6 weeks, the speed-accuracy-centered approach was verified to be significant clinically with extensive applicability [56]. Similarly, self-administered smart tablet therapy is an encouraging option to optimize everyday life communication in anomia cases, particularly in settings where SLPs are inadequate for chronic PSA, hence validating the efficiency of utilizing smart tablets to advance naming in PSA [3, 57, 58]. Another study that applied 1-h dose of computer-delivered script practice with and without a break authenticated the probability learning facilitation. However, optimal dosage and scheduling parameters are a topic for prospective analysis [59].

Based on 278 cases attending self-managed computerized speech and language therapy (CSLT) in the subacute phase, CSLT plus usual care rendered a clinically notable advancement in relevant word-finding correlated to the conversation. Generating a demand to couple CSLT with SLT as demonstrated to be more productive than no-therapy [60]. Writing practices can be affected in aphasia and resume less well than other language modalities. Assistive technology, such as speech-to-text software, can be used in therapeutics to counterbalance writing impairments [61].

Telerehabilitation is a novel rehabilitation procedure that comfortably permits victims to undergo therapy at home, bypassing the practitioner's need to move. Multimodal language therapy performed through synchronized telerehabilitation illustrated positive impressions on chronic PSA functional communication [26, 48]. Likewise, telespeech therapy alleviates the remoteness obstacle and the influence of mobility difficulties appending PSA management. Recent research completed in Canada prompts other debates by showing that multimodal language treatment performed by synchronized telespeech therapy yielded good outcomes on functional language in CVA cases [26].

\section{Pharmacotherapy}

Several clinical studies have scrutinized the potency of diverse pharmacological options for PSA language restoration. Pieces of evidence advocating pharmacological stimulation of neuroplasticity are still limited. Furthermore, the contemporary guidelines suggest drug coupled with SLT on a case-based consideration, yet register a shortage of distinct regimen recommendations. Nevertheless, pharmacotherapy appears to bear a promising strategy for ameliorating PSA. Recently, Berthier and colleagues pointed out ten reasons for advocating future PSA pharmacotherapy studies. Generally, based on previous studies, pharmacotherapy bears the advantages of better safety and tolerability, speeding PSA recovery rate, and better results even when unpaired with other therapeutic options reported. Further, augmentation of other aphasia therapy, improvement of the cognitive and 
behavioral deficit, and effectivity after drug withdrawal were reported [47, 62].

Stroke interruption of neurotransmitter conduits justifies the rationale for PSA pharmacotherapy intervention. The pathways affected most are those connecting the brainstem and forebrain to the cortical language regions and deep gray nuclei. Hence, pharmacological modulation of these neurotransmitter pathways tends to facilitate synaptic plasticity resulting in PSA improvement [63].

Amphetamine (AMPH) is a stimulant drug, which acts by promoting monoamines' synaptic concentration. Animal studies on AMPH propose a two-phase mechanism, the first phase centered on the resolution of diaschisis and a second phase contributing to neuronal remodeling. Anatomically, data suggested that cortico-efferent plasticity of axonal sprouting contributes to improved motor recovery. Dextroamphetamine is a catecholaminergic drug, the mechanism focused on the dopamine and norepinephrine reuptake inhibition. The receptor pharmacology of the $\mathrm{D}$-amphetamine is not yet entirely clear. In animal studies, it has been observed to enhance neural sprouting and synaptogenesis post infarction. In animal study designs, amphetamine coupled with behavioral therapies were experimented for language neuromodulation. Likewise, Donepezil, coupled with dextroamphetamine sulfate, has been examined for recovery augmentation and safety, with the evidence that this combination was well-tolerated without unfavorable cardiac outcomes (pulse rate and blood pressure). Lack of mood evaluation, which is a vital component when evaluating the consequences of stimulants, and the absence of specific stipulation on higher-dose side effects were the notable drawbacks to the evidence [64].

Atomoxetine (ATX) is a selective noradrenaline (NA) reuptake inhibitor. Recent studies link ATX to brain plasticity in humans [65]. Yamada and colleagues, in 2016, confirmed that atomoxetine treatment was safe and achievable for PSA victims. Following titration of $40 \mathrm{mg}, 80 \mathrm{mg}$, and $120 \mathrm{mg}$ of atomoxetine at 2 weeks before admission, 1 week before admission, and 13 days of hospitalization, respectively, and combined with intensive SLT, pressing demands for prospective trials of ATX coupled with NIBS are suggested [66].

ATX mechanism is the center in increasing NA levels throughout the cerebral cortex in response to high NA transporter (NAT) levels in the cortical regions. Further, ATX proved to increases dopamine levels in the frontal lobe. ATX has additional abilities such as antagonistic effects on the $N$-methyl-D-aspartate receptors (NMDA Rs) and beneficial effects in some in vitro studies. Nevertheless, animal studies showed that pretreatment with ATX facilitates inhibition of ischemia-induced excitotoxicity post cerebral ischemic injury. SPECT studies in
PSA patients verified an increase in blood flow across the perisylvian speech zone coupled with intensive SLT $[65,66]$.

Comparably, Zhang and colleagues, in 2018, supported that donepezil (DPZ) has a striking impact in improving acoustic comprehension, naming, word reproduction, and oral narration; comparatively, memantine has a vital impact in promoting naming ability, impromptu speech, and word reproduction [67]. Nevertheless, several studies reported a negative impact of DPZ on aphasia therapy $[63,68,69]$.

Memantine is a non-competitive NMDA-receptor antagonist with approval for Alzheimer's disease treatment. In contrast, DPZ is classified as a cholinergic drug. Emerging evidence links the cholinergic activity of DPZ with the left temporal lobe, hence enhancing language processes, especially posterior cerebral lesions. Further, cholinergic drugs enhance cognitive functions thought to play a role in language enhancement post-stroke [63, 70, 71].

Animal studies exhibited a decrease in post-stroke catecholamine levels in the cortical regions, leading to delays in stroke recovery. Levodopa is a catecholamine drug. A specific role of catecholamine for language functions is theorized on catecholaminergic system simulations. Augmentation of attentional regions that drive language output is the principal outcome. Piracetam is a nonspecific GABA derivative classified as a nootropic drug. The specific mechanisms specific to piracetam is unknown.

Nevertheless, the proposed mechanism focused on facilitating cholinergic neurotransmitters and increasing regional cerebral blood flow effects. Excitation of neurotransmission on amines was also thought. Similarly, bromocriptine is the most studied drug for PSA. Dopaminergic agents are classified as D2 agonists and proved to bear a significant number of contraindications [62, 63].

The importance of some of these drugs (bromocriptine, piracetam, and levodopa) in PSA repairs is still unsatisfactory and unsettled. Notwithstanding, on a case basis, drug therapy recommended by the current guidelines are proposing the necessity for thorough studies on the dosage and timing before officiating regular use recommendations [47, 63, 67, 72].

For many years in Asian countries, acupuncture and traditional herbal remedies bore a favorable practice for PSA management and other post-stroke complications. However, the precise mechanism of acupuncture for aphasia therapy is unknown. There are several techniques to apply acupuncture. These include traditional acupuncture (TA), Jin's three needles (insertion of 3 needles on vital acupoints), and electroacupuncture. Other methods involve scalp acupuncture (SA) in which 
needles excite various scalp regions for particular nerve reflexively and tongue acupuncture (acupuncture needle inserted and twisted in tongue acupoints) [73-75].

One electroacupuncture study applied the current intensity of $2 \mathrm{~mA}$ to arouse the Tongli acupoint ( $1 \mathrm{in}$. over the transverse crease of the wrist). The Xuan Zhong acupoint ( $3 \mathrm{in}$. beyond the top of the external malleolus) was also stimulated. Hence, the study exhibited that acupuncture points could provoke multiple cerebral language areas in Broca's aphasia cases, which may bear a beneficial effect on PSA recovery [75]. Furthermore, one of the earliest studies summarizing current PSA evidence of acupuncture, covering twenty-eight RCTs in $>1700$ cases, presented proof on the acupuncture efficacy in promoting PSA functional communication and language role. However, the absence of distinct time points poststroke, and acupuncture variety classification was a notable study drawback [76].

However, another meta-analysis proved that SA, tongue acupuncture, and Jin's 3-needle acupuncture could better improve PSA than TA alone or coupled with rehabilitation training. Similar findings for tongue acupuncture were reported by $\mathrm{Wu}$ and colleagues 3 years prior. Nevertheless, the quality of the incorporated studies was inadequate [74, 77].

Among the oriental herbal medicine, Jie Yu Dan (JYD) has been recently studied for PSA. Other herbal remedies previously studied for aphasia include Kang Yu Tang, Dan Xi Tang, and Niu Huang. The suggested mechanism of JYD on PSA concentrates on neuroregeneration. Moreover, post-stroke reduction of oxidative stress and inflammation for neurogenesis is thought. Furthermore, other JYD positive effects include enhancing blood flow in ischemic lesions, promoting microcirculation, boosting blood circulation, and forcing collateral circulation. A recent meta-analysis of $10 \mathrm{RCTs}$ proposing moderate supportive testimony of a four-week course of treatment of JYD bears positive effects on PSA inconsiderate of the onset with better safety profile when coupled with PSA conventional therapy. However, the study had all RCTs including only Chinese people, which does not reflect the effectiveness of other ethnicities, yet tiny subjects were enrolled [73].

Stem cell, cellular, and gene therapies represent a fundamental transformation in the therapeutical paradigm in chronic pathologies owing to its multi-cellular molecules and pathways resembling physiological mechanisms. Over the past 20 years, mesenchymal stem cells (MSCs) from the umbilical cord blood, bone marrow $(\mathrm{BM})$, and adipose tissue were employed in post-stroke cases via intravenous (IV) and intra-arterial routes. Practice incorporated intrathecal $(\mathrm{I} / \mathrm{T})$ and intracerebral routes as well. Hypothesized mechanisms focus on the enhancement of neurotrophic growth factor secretion by stem cells, hence facilitating recovery. Furthermore, emerging proof advocates the role of neuronal circuit reestablishment, by increasing nerve fiber sprouting, and diminished apoptosis. Nevertheless, the purpose of decreased peri-infarct inflammation and angiogenesis was highlighted $[78,79]$.

Most recently, IV and I/T administrations of CD271 ${ }^{+}$ stem cells at a single dosage of $2-5 \times 106$ cells $/ \mathrm{kg}$ were established as a safe alternative with excellent outcomes in managing CVA cases. The same effect was observed for chronic spasticity. Nevertheless, a tiny number of cases (8 patients) was a significant limitation to this study. On the contrary, another research on BM-derived mononuclear cells (BM-MNCs), which enrolled 39 SVA patients in which none received IV alteplase in the acute phase, confirmed the safety profile. However, no significant enhancement of the motor, PSA, or infarction volume was detected. Furthermore, aphasia and a convulsion secondary to intracerebral hemorrhage were reported as the complexity of genetically altered allogeneic MSCs 3 years post-implantation [78-80].

\section{Music-based therapy}

Melodic intonation therapy (MIT) focuses on the application of musical components of speech (rhythmic and melodic) to enhance language composition with contralesion cerebral proficient language areas involved. There is assuring proof from one small, pilot RCT of 20 PSA sufferers that MIT can impact the communication abilities of stroke victims with Broca's aphasia; generally, RCT-based effectiveness data is still limited [81]. Similarly, Slavin and colleagues showed a prosperous MIT case-based research on a 10-year patient post-PSA. Initially, this patient was offered SLT and a modified MIT procedure. Registering that a 9-month MIT combined with musical components and coupled with group treatment may result in augmented speech and motor language abilities [82]. Further, there is assuring testimony that adjustments to home-based applications, such as aphasia-favorable written guidance and music, promote proper home training [83].

\section{Psychosocial interventions}

In one systematic paper, based on seventy analysis studies, the likelihood of post-stroke victims creating social chains, having a friendship relationship, and support aided defeating PSD manifestations [84]. Likewise, the communication partner training $(\mathrm{CPT})$ is a vital modifier of the connection linking the participation and brain; therefore, CPT should approach communicative participation throughout the rehabilitation to strongly reintegrate into their everyday post-stroke community experiences [85]. 
Earlier research has registered that CPT enhances and tackles the adverse psychosocial outcomes for PSA victims and communication partners. In a survey concerning 122 SLPs with expertise in PSA management, the majority proclaimed managing a variety of post-stroke communication dysfunctions. Consequently, approaches to promote $\mathrm{CPT}$ should center on auditing CPT performance, fixing it into policies. Further, when CPT coaching is unobstructedly available with widespread acceptance, it will set an imperative element of stroke rehabilitation [86]. In contrast, in the most contemporary online survey, research indicated 50\% of PSA victims' family affiliates' dissatisfaction with the PSA knowledge and did not remember getting information from the clinicians; therefore, health professionals are warranted to support better the knowledge demands of family members in a written form and via discussions, throughout the initial times of care [87].

Wang and colleagues validated the significance of immediate and efficient depression screening, therapy, and stroke rehabilitation and appeared to be fundamental for recovery and conquering the post-stroke burden among the survivors. Further, at 90 days follow up, unlike PSA, there is a precise connection between physical independence and PSD, gender, and stroke intensity [88]. When contrasted to conventional psychological mediations, the E-mental health (EMH) grants useful and cost-effective mediations that can encompass a broad community which hence forms an encouraging therapy modality for the following decade. Despite meeting the bulk of the overall evaluation and aphasia-specific evaluation standards, it still needs ample redevelopment to be valuable to PSA victims [27].

Stepped psychological care offers a regular assessment and mediations for psychological predicaments in a structure of four levels for preventing and managing depression among aphasia cases, whereby level 1 incorporates cases without clinically notable depression and mild depression manifestations benefit from level 2 mediations. In contrast, PSA cases with depression necessitating specialist intervention (moderate to severe) befall in levels 3 and 4, yet, knowledge breaks on methodological shortcomings and consolidating stepped psychological mediation crosswise the continuum prevails [89]. Marshall and colleagues reported the usefulness of the emerging of the mindfulness meditation bearing benefits of a low-cost foundation, quick explanation of method to patients, and capability for extensive application in clinical usage as a complement to the current languagefocused mediations [90].

In the community aspect, the gap amid approaches applied by community-based exercise programs and those suggested in the research (written documents vs. verbal approaches) and the shortage of equipped staff to manage and communicate with PSA victims are still often recognized as a hindrance to grant access [91]. Eye movement desensitization and reprocessing (EMDR) (Fig. 1) offers, among other techniques, alternating bilateral stimulation. The novel findings presented by Guina and colleagues through a case-based research of a lady in her fifties with PSA-PSD comorbidity marks EMDR as an ideal treatment modality for PSA cases with psychiatric pathologies [92].

\section{Limitations and future research directions}

While evaluating the studies reported in this review, there are some limitations to consider. These include the following: (i) some studies of low quality were incorporated as all assembled articles published in the time frame highlighted in the methodology were reported; (ii) studies with a small number of participants (case reports) were included; (iii) the researches which give positive results were prioritized in obtaining some conclusions; (iv) some reported studies performed only in a single ethnic group (herbal remedies); and (v) incorporated studies may have diverse therapeutic protocols.

Recent neuroimaging studies link PSA recovery with the neuronal reorganization. Future clinical studies are warranted to better harness neuroplasticity in the administration of CVA and SVA therapy to increase language and communication recovery. Further, as emerging initiatives combining therapies are observed, future studies in large samples are expected to explore the optimal dose and timing of different therapeutic modalities and their role when offered in combination. Furthermore, the concern about the tolerability and safety of drug options such as AMPH and long-term effects of the combined treatment in ATX is a matter to be approached in future researches.

Nevertheless, the emerging NIBS modalities such as spinal cord in language processing remain controversial, owing to speculative mechanisms over the corticospinal system. A sufficient number of cases encompass different lesion parameters (unilateral LH lesions) warranted to declare equivalent beneficial effects and establish the spinal cord's role in language processing.

However, as SLT is overwhelmed by insufficient data on the dosage and intensity, future research investigating optimal dosage and scheduling parameters is obliged. Lastly, the forthcoming of E-mental health programs should be explicitly advanced for PSA cases by incorporating end-users in the conception and advancement process to guarantee usability.

\section{Conclusions}

There is marked progress in the management of subacute and chronic PSA. NIBS, coupled with SLT, is more efficient in promoting the recovery means than 
The management options for Subacute and Chronic Vascular Aphasia

Subacute/Chronic

Vascular Aphasia

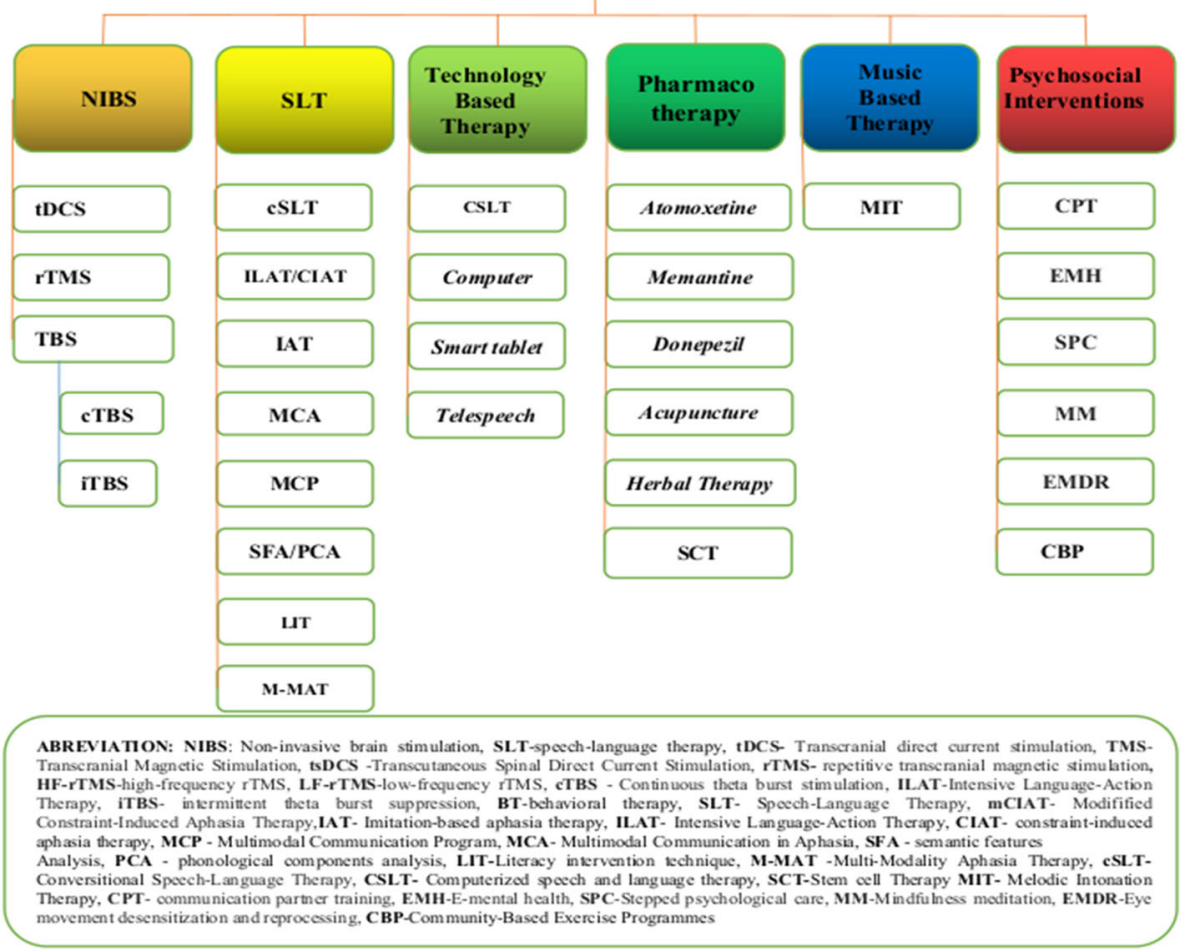

Fig. 1 Compiling six major choices and 30 sub-options for management of chronic and subacute vascular aphasia based on English studies from PubMed search (2016 through 2020), the last row highlights the abbreviated terminologies

monotherapy. Among NIBS modalities, rTMS is safe and exhibiting advantage of effectiveness for both SVA and CVA, but less cost-effective. The debate on rTMS frequency authenticates proof on LF-rTMS efficacy than the high-frequency rTMS, yet clinical significance levels are the matter of future determination. tsDCS and cerebellar tDCS are promising novel NIBS tools requiring further researches.

SLT is the cornerstone of subacute and chronic PSA management, yet the optimal dosage of the emerging high-intensity therapies is controversial. Technologybased and telespeech therapies mark PSA treatment's future due to low operating costs, alleviating the remoteness obstacle, particularly in the setting where SLPs are inadequate for chronic PSA. As the current guideline recommends pharmacotherapy coupled with SLT on a case basis, no SVA and CVA medications are currently approved.

Throughout the continuum of care, approximately $50 \%$ of PSA victims' family affiliates report dissatisfaction with the PSA knowledge given postdischarge. EMH, EMDR, creating social chains, having a friendship relationship, and CPT provision are the most useful approaches in combating PSA-PSD comorbidity, forming around $60 \%$ of CVA cases. Future large, multiethnic, and multicenter RCTs focusing on the neuroplasticity of the CVA and SVA therapies are warranted. Further, studies exploring the optimal dose and timing of different therapeutic modalities and addressing tolerability and safety of drug options are necessitated.

\section{Abbreviations}

PSA: Post-stroke aphasia; rTMS: Repetitive transcranial magnetic stimulation: SLPs: Speech-language pathologists; PSD: Post-stroke depression;

SVA: Subacute vascular aphasia; CVA: Chronic vascular aphasia; IFG: Inferior frontal gyrus; M-MAT: Multimodal aphasia treatment; ICAPs: Comprehensive aphasia programs; NIBS: Noninvasive brain stimulation; SLT: Speech-language therapy; tDCS: Transcranial direct current stimulation; TMS: Transcranial magnetic stimulation; tsDCS: Transcutaneous spinal direct current stimulation; HF-rTMS: High-frequency rTMS; LF-rTMS: Low-frequency rTMS; CTBS: Continuous theta burst stimulation; ILAT: Intensive language-action therapy; iTBS: Intermittent theta burst suppression; BT: Behavioral therapy; mCIAT: Modified constraint-induced aphasia therapy; IAT: Imitation-based 
aphasia therapy; CIAT: Constraint-induced aphasia therapy; MCP: Multimodal communication program; MCA: Multimodal communication in aphasia; SFA: Semantic features analysis; PCA: Phonological components analysis; LIT: Literacy intervention technique; CSLT: Conversational speech-language therapy; CSLT: Computerized speech and language therapy; MIT: Melodic intonation therapy; CPT: Communication partner training; EMH: E-mental health; SPC: Stepped psychological care; MM: Mindfulness meditation; EMDR: Eye movement desensitization and reprocessing; CBP: Communitybased exercise programs; DPZ: Donepezil; ATX: Atomoxetine; TA: Traditional acupuncture; SA: Scalp acupuncture; JYD: Jie Yu Dan; MSCs: Mesenchymal stem cells; BM-MNCs: Bone marrow-derived mononuclear cells

\section{Acknowledgements}

The authors thank editorial team and two anonymous reviewers for their valuable advice regarding improvements in a previous version of this review.

\section{Authors' contributions}

Authors contributed to this paper, with the design (SSM), literature search (SSM), drafting (SSM), revision (SSM and XC), and editing (SSM and XC). The authors read and approved the final manuscript.

\section{Funding}

Not applicable

\section{Availability of data and materials}

Not applicable

\section{Ethics approval and consent to participate}

Not applicable

\section{Consent for publication}

Not applicable

\section{Competing interests}

The authors declare that they have no competing interests.

\section{Author details}

'Department of Neurology, The Clinical Medicine School of Yangtze University, The First Affiliated Hospital of Yangtze University, Jingzhou 424023, Hubei, China. ${ }^{2}$ Mirembe National Mental Health Hospital, Dodoma, Tanzania. ${ }^{3}$ Department of Neurology and Rehabilitation, The First Affiliated Hospital of Yangtze University, Jingzhou, Hubei, China.

Received: 15 July 2020 Accepted: 24 August 2020

Published online: 04 September 2020

\section{References}

1. Marangolo P, Fiori V, Shofany J, Gili T, Caltagirone C, Cucuzza G, et al. Moving beyond the brain: transcutaneous spinal direct current stimulation in post-stroke aphasia. Front Neurol. 2017;8:400.

2. Dionísio A, Duarte IC, Patrício M, Castelo-Branco M. Transcranial magnetic stimulation as an intervention tool to recover from language, swallowing and attentional deficits after stroke: a systematic review. Cerebrovasc Dis. 2018:46(3-4):178-85.

3. Lavoie M, Bier N, Macoir J. Efficacy of a self-administered treatment using a smart tablet to improve functional vocabulary in post-stroke aphasia: a caseseries study. Int J Lang Commun Disord. 2019;54(2):249-64.

4. Kim G, Min D, Lee EO, Kang EK. Impact of co-occurring dysarthria and aphasia on functional recovery in post-stroke patients. Ann Rehabil Med 2016;40(6):1010-7.

5. Buijck B. Ribbers G. The challenges of nursing stroke management in rehabilitation centres: Springer International Publishing; 2018.

6. Bernhardt J, Hayward KS, Kwakkel G, Ward NS, Wolf SL, Borschmann K, et al. Agreed definitions and a shared vision for new standards in stroke recovery research: The Stroke Recovery and Rehabilitation Roundtable taskforce. Int J Stroke. 2017;12(5):444-50.

7. Tippett DC, Hillis AE. Vascular aphasia syndromes. In: Neurobiology of Language. Academic Press;2016.p. 913-922.

8. El-Tallawy HN, Gad AH, Ali AM, Abd-El-Hakim MN. Relative frequency and prognosis of vascular aphasia (follow-up at 3 months) in the Neurology
Department of Assiut University Hospital. The Egyptian Journal of Neurology, Psychiatry and Neurosurgery. 2019:55(1):41.

9. Lucchese G, Pulvermüller F, Stahl B, Dreyer FR, Mohr B. Therapy-induced neuroplasticity of language in chronic post stroke aphasia: a mismatch negativity study of (a)grammatical and meaningful/less mini-constructions. Front Hum Neurosci. 2017;10:669.

10. Mohr B, MacGregor LJ, Difrancesco S, Harrington K, Pulvermüller F, Shtyrov Y. Hemispheric contributions to language reorganisation: an MEG study of neuroplasticity in chronic post stroke aphasia. Neuropsychologia. 2016;93(Pt B):413-424.

11. Gerstenecker A, Lazar RM. Language recovery following stroke. Clin Neuropsychol. 2019;33(5):928-47.

12. Ballester BR, Maier M, Duff A, Cameirão M, Bermúdez S, Duarte $E$, et al. A critical time window for recovery extends beyond one-year post-stroke. J Neurophysiol. 2019:122(1):350-7.

13. Mohr B. Neuroplasticity and functional recovery after intensive language therapy in chronic post stroke aphasia: Which factors are relevant? Front Hum Neurosci. 2017;11:332.

14. Robson H, Griffiths TD, Grube M, Woollams AM. Auditory, phonological, and semantic factors in the recovery from Wernicke's aphasia poststroke: predictive value and implications for rehabilitation. Neurorehabil Neura Repair. 2019;33(10):800-12.

15. Kiran S, Thompson CK. Neuroplasticity of language networks in aphasia: advances, updates, and future challenges. Front Neurol. 2019;10:295.

16. Marebwa BK, Fridriksson J, Yourganov G, Feenaughty L, Rorden C, Bonilha L. Chronic post-stroke aphasia severity is determined by fragmentation of residual white matter networks. Sci Rep. 2017;7(1):8188.

17. Grechuta K, Bellaster BR, Munne RE, Bernal TU, Hervas BM, Segundo RS, et al The effects of silent visuomotor cueing on word retrieval in Broca's aphasics: a pilot study. IEEE Int Conf Rehabil Robot. 2017;2017:193-9.

18. Norise $\mathrm{C}$, Hamilton $\mathrm{RH}$. Non-invasive brain stimulation in the treatment of post-stroke and neurodegenerative aphasia: parallels, differences, and lessons learned. Front Hum Neurosci. 2017;10:675.

19. Sebastian R, Saxena S, Tsapkini K, Faria AV, Long C, Wright A, et al. Cerebellar tDCS: a novel approach to augment language treatment poststroke. Front Hum Neurosci. 2017:10:695.

20. Harvey DY, Podell J, Turkeltaub PE, Faseyitan O, Coslett HB, Hamilton RH. Functional reorganization of right prefrontal cortex underlies sustained naming improvements in chronic aphasia via repetitive transcranial magnetic stimulation. Cogn Behav Neurol. 2017:30(4):133-44.

21. Spielmann K, van de Sandt-Koenderman WM, Heijenbrok-Kal MH, Ribbers GM. Comparison of two configurations of transcranial direct current stimulation for aphasia treatment. J Rehabil Med. 2018;50(6):527-33.

22. Hu XY, Zhang T, Rajah GB, Stone C, Liu LX, He JJ, et al. Effects of different frequencies of repetitive transcranial magnetic stimulation in stroke patients with non-fluent aphasia: a randomized, sham-controlled study. Neurol Res. 2018;40(6):459-65.

23. Stahl B, Mohr B, Büscher $V$, Dreyer FR, Lucchese G, Pulvermüller F. Efficacy of intensive aphasia therapy in patients with chronic stroke: a randomised controlled trial. J Neurol Neurosurg Psychiatry. 2018;89(6):586-92.

24. Sekhon JK, Oates J, Kneebone I, Rose M. Counselling training for speechlanguage therapists working with people affected by post-stroke aphasia: a systematic review. Int J Lang Commun Disord. 2019:54(3):321-46.

25. Wray F, Clarke D, Forster A. Post-stroke self-management interventions: a systematic review of effectiveness and investigation of the inclusion of stroke survivors with aphasia. Disabil Rehabil. 2018;40(11):1237-51.

26. Macoir J, Sauvageau VM, Boissy P, Tousignant M, Tousignant M. In-home synchronous telespeech therapy to improve functional communication in chronic poststroke aphasia: results from a quasi-experimental study. Telemed J E Health. 2017;23(8):630-9.

27. Clunne SJ, Ryan BJ, Hill AJ, Brandenburg C, Kneebone I. Accessibility and applicability of currently available e-mental health programs for depression for people with poststroke aphasia: scoping review. J Med Internet Res. 2018;20(12):e291.

28. Heikkinen PH, Pulvermüller F, Mäkelä JP, Ilmoniemi RJ, Lioumis P, Kujala T, et al. Combining rTMS with intensive language-action therapy in chronic aphasia: a randomized controlled trial. Front Neurosci. 2019;12:1036.

29. Biou E, Cassoudesalle H, Cogné M, Sibon I, De Gabory I, Dehail P, et al. Transcranial direct current stimulation in post-stroke aphasia rehabilitation: a systematic review. Ann Phys Rehabil Med. 2019:62(2):104-21. 
30. Vila-Nova C, Lucena PH, Lucena R, Armani-Franceschi G, Campbell FQ. Effect of anodal tDCS on articulatory accuracy, word production, and syllable repetition in subjects with aphasia: a crossover, double-blinded, shamcontrolled trial. Neurol Ther. 2019;8(2):411-24.

31. Bucur M, Papagno C. Are transcranial brain stimulation effects long-lasting in post-stroke aphasia? A comparative systematic review and meta-analysis on naming performance. Neurosci Biobehav Rev. 2019;102:264-89.

32. Cirillo G, Di Pino G, Capone F, Ranieri F, Florio L, Todisco V, et al. Neurobiological after-effects of non-invasive brain stimulation. Brain Stimul. 2017;10(1):1-18

33. Norise C, Sacchetti D, Hamilton R. Transcranial direct current stimulation in post-stroke chronic aphasia: the impact of baseline severity and task specificity in a pilot sample. Front Hum Neurosci. 2017;11:260.

34. Meinzer M, Darkow R, Lindenberg R, Flöel A. Electrical stimulation of the motor cortex enhances treatment outcome in post-stroke aphasia. Brain 2016;139(Pt 4):1152-63.

35. Woodhead ZVJ, Kerry SJ, Aguilar OM, Ong YH, Hogan JS, Pappa K, et al. Randomized trial of iReadMore word reading training and brain stimulation in central alexia. Brain. 2018;141(7):2127-41.

36. Rosso C, Arbizu C, Dhennain C, Lamy JC, Samson Y. Repetitive sessions of tDCS to improve naming in post-stroke aphasia: insights from an individual patient data (IPD) meta-analysis. Restor Neurol Neurosci. 2018;36(1):107-16.

37. Carlson HL, Jadavji Z, Mineyko A, Damji O, Hodge J, Saunders J, et al. Treatment of dysphasia with rTMS and language therapy after childhood stroke: multimodal imaging of plastic change. Brain Lang. 2016;159:23-34.

38. Lefaucheur JP, Aleman A, Baeken C, Benninger DH, Brunelin J, Di Lazzaro V, et al. Evidence-based guidelines on the therapeutic use of repetitive transcranial magnetic stimulation (rTMS): an update (2014-2018). Clin Neurophysiol. 2020;131(2):474-528.

39. Zhang H, Chen $Y$, Hu R, Yang L, Wang M, Zhang J, et al. rTMS treatments combined with speech training for a conduction aphasia patient: a case report with MRI study. Medicine (Baltimore). 2017;96(32):e7399.

40. Georgiou A, Konstantinou N, Phinikettos I, Kambanaros M. Neuronavigated theta burst stimulation for chronic aphasia: two exploratory case studies. Clin Linguist Phon. 2019;33(6):532-46.

41. Harvey DY, Mass JA, Shah-Basak PP, Wurzman R, Faseyitan O, Sacchetti DL, et al. Continuous theta burst stimulation over right pars triangularis facilitates naming abilities in chronic post-stroke aphasia by enhancing phonological access. Brain Lang. 2019;192:25-34.

42. Griffis JC, Nenert R, Allendorfer JB, Szaflarski JP. Interhemispheric plasticity following intermittent theta burst stimulation in chronic poststroke aphasia. Neural Plast. 2016;2016:4796906.

43. Gleichgerrcht E, Fridriksson J, Rorden C, Nesland T, Desai R, Bonilha L. Separate neural systems support representations for actions and objects during narrative speech in post-stroke aphasia. Neuroimage Clin. 2015;10: 140-5.

44. Kjellén E, Laakso K, Henriksson I. Aphasia and literacy-the insider's perspective. Int J Lang Commun Disord. 2017;52(5):573-84.

45. Preisig BC, Eggenberger N, Cazzoli D, Nyffeler T, Gutbrod K, Annoni JM, et al. Multimodal communication in aphasia: perception and production of co-speech gestures during face-to-face conversation. Front Hum Neurosci. 2018;12:200

46. Dignam J, Copland D, Rawlings A, O'Brien K, Burfein P, Rodriguez AD. The relationship between novel word learning and anomia treatment success in adults with chronic aphasia. Neuropsychologia. 2016;81:186-97.

47. Winstein CJ, Stein J, Arena R, Bates B, Cherney LR, Cramer SC, et al. American Heart Association Stroke Council, Council on Cardiovascular and Stroke Nursing, Council on Clinical Cardiology, and Council on Quality of Care and Outcomes Research. Guidelines for adult stroke rehabilitation and recovery: a guideline for healthcare professionals from the American Heart Association/American Stroke Association. Stroke. 2016;47(6):e98-e169.

48. Palmer R, Witts $H$, Chater T. What speech and language therapy do community dwelling stroke survivors with aphasia receive in the UK? PLoS One. 2018;13(7):e0200096.

49. Hoover EL, Caplan DN, Waters GS, Carney A. Communication and quality of life outcomes from an interprofessional intensive, comprehensive, aphasia program (ICAP). Top Stroke Rehabil. 2017;24(2):82-90.

50. Rose ML, Copland D, Nickels L, Togher L, Meinzer M, Rai T, et al. Constraintinduced or multi-modal personalized aphasia rehabilitation (COMPARE): a randomized controlled trial for stroke-related chronic aphasia. Int J Stroke. 2019;14(9):972-6.
51. Duncan ES, Small SL. Imitation-based aphasia therapy increases narrative content: a case series. Clin Rehabil. 2017;31(11):1500-7.

52. Pierce JE, Menahemi-Falkov M, O'Halloran R, Togher L, Rose ML. Constraint and multimodal approaches to therapy for chronic aphasia: a systematic review and meta-analysis. Neuropsychol Rehabil. 2019;29(7):1005-41.

53. Kaviani S, Samaei A, Salmani M, Ansari NN, Dehnavi F, Shahverdi E. Effects of multimodal communication program on patients with chronic aphasia: a single-subject A-B-A design study. Electron Physician. 2018;10(3):6439-47.

54. Russo MJ, Prodan V, Meda NN, Carcavallo L, Muracioli A, Sabe L, et al. Hightechnology augmentative communication for adults with post-stroke aphasia: a systematic review. Expert Rev Med Devices. 2017;14(5):355-70.

55. Flöel A. Computerised speech and language therapy in post-stroke aphasia. Lancet Neurol. 2019;18(9):806-7.

56. Conroy P, Sotiropoulou Drosopoulou C, Humphreys GF, Halai AD, Lambon Ralph MA. Time for a quick word? The striking benefits of training speed and accuracy of word retrieval in post-stroke aphasia. Brain. 2018;141(6): 1815-27.

57. Kurland J, Liu A, Stokes P. Effects of a tablet-based home practice program with telepractice on treatment outcomes in chronic aphasia. J Speech Lang Hear Res. 2018;61(5):1140-56.

58. Godlove J, Anantha V, Advani M, Des Roches C, Kiran S. Comparison of therapy practice at home and in the clinic: a retrospective analysis of the Constant Therapy Platform data set. Front Neurol. 2019;10:140.

59. Cherney LR, Braun EJ, Lee JB, Kocherginsky M, Van Vuuren S. Optimising recovery in aphasia: learning following exposure to a single dose of computer-based script training. Int J Speech Lang Pathol. 2019;21(5):448-58.

60. Palmer R, Dimairo M, Latimer N, Cross E, Brady M, Enderby P, et al. Computerised speech and language therapy or attention control added to usual care for people with long-term post-stroke aphasia: the Big CACTUS three-arm RCT. Health Technol Assess. 2020;24(19):1-176.

61. Marshall J, Caute A, Chadd K, Cruice M, Monnelly K, Wilson S, et al. Technology-enhanced writing therapy for people with aphasia: results of a quasi-randomized waitlist controlled study. Int J Lang Commun Disord. 2019:54(2):203-20.

62. Berthier ML. Ten key reasons for continuing research on pharmacotherapy for post-stroke aphasia. Aphasiology. 2020;31:1-35.

63. Saxena $\mathrm{S}$, Hillis AE. An update on medications and noninvasive brain stimulation to augment language rehabilitation in post-stroke aphasia. Expert Rev Neurother. 2017;17(11):1091-110.

64. Walker-Batson D, Mehta J, Smith P, Johnson M. Amphetamine and other pharmacological agents in human and animal studies of recovery from stroke. Prog Neuropsychopharmacol Biol Psychiatry. 2016;64:225-30.

65. Ward A, Carrico C, Powell E, Westgate PM, Nichols L, Fleischer A, et al. Safety and improvement of movement function after stroke with atomoxetine: a pilot randomized trial. Restor Neurol Neurosci. 2017;35(1):1-10.

66. Yamada N, Kakuda W, Yamamoto K, Momosaki R, Abo M. Atomoxetine administration combined with intensive speech therapy for post-stroke aphasia: evaluation by a novel SPECT method. Int J Neurosci. 2016;126(9):829-38.

67. Zhang X, Shu B, Zhang D, Huang L, Fu Q, Du G. The efficacy and safety of pharmacological treatments for post-stroke aphasia. CNS Neurol Disord Drug Targets. 2018;17(7):509-21.

68. Berube $\mathrm{S}$, Hillis AE. Advances and innovations in aphasia treatment trials. Stroke. 2019;50(10):2977-84.

69. Woodhead ZV, Crinion J, Teki S, Penny W, Price CJ, Leff AP. Auditory training changes temporal lobe connectivity in 'Wernicke's aphasia': a randomised trial. J Neurol Neurosurg Psychiatry. 2017;88(7):586-94.

70. Llano DA, Small SL. Pharmacotherapy for aphasia. In:Neurobiology /of Language. Academic Press;2016.p.1067-1083.

71. Carrillo-Mora P, Alcantar-Shramm JM, Almaguer-Benavides KM, MacíasGallardo JJ, Fuentes-Bello A, Rodríguez-Barragán MA. Pharmacological stimulation of neuronal plasticity in acquired brain injury. Clin Neuropharmacol. 2017;40(3):131-9.

72. Zhang J, Wei R, Chen Z, Luo B. Piracetam for aphasia in post-stroke patients: a systematic review and meta-analysis of randomized controlled trials. CNS Drugs. 2016;30(7):575-87

73. Zhang T, Wu X, Cao S, Park S. Efficacy of the Oriental herbal medicine, Jie Yu Dan, for alleviating post-stroke aphasia: a systematic review and metaanalysis of randomized clinical trials. European Journal of Integrative Medicine. 2018;24:35-48.

74. Tang HY, Tang W, Yang F, Wu WW, Shen GM. Efficacy of acupuncture in the management of post-apoplectic aphasia: a systematic review and meta- 
analysis of randomized controlled trials. BMC complementary and alternative medicine. 2019;19(1):282.

75. Chang J, Zhang H, Tan Z, Xiao J, Li S, Gao Y. Effect of electroacupuncture in patients with post-stroke motor aphasia. Wiener klinische Wochenschrift. 2017;129(3-4):102-9

76. Zhang B, Han Y, Huang X, Liu Z, Li S, Chang J, et al. Acupuncture is effective in improving functional communication in post-stroke aphasia: a systematic review and meta-analysis of randomized controlled trials. Wien Klin Wochenschr. 2019;131(9-10):221-232.

77. Wu Q, Hu X, Wen X, Li F, Fu W. Clinical study of acupuncture treatment on motor aphasia after stroke. Technology and Health Care. 2016;24(s2):S691-6.

78. Stancioiu F, Papadakis GZ, Lazopoulos G, Spandidos DA, Tsatsakis A, Floroiu M, Badiu C. CD271+ stem cell treatment of patients with chronic stroke. Experimental and Therapeutic Medicine. 2020 Sep 1;20(3):2055-62.

79. Ghali AA, Yousef MK, Ragab OA, ElZamarany EA. Intra-arterial infusion of autologous bone marrow mononuclear stem cells in subacute ischemic stroke patients. Frontiers in Neurology. 2016;7:228.

80. Scott RM. De novo arteriovenous malformation growth secondary to implantation of genetically modified allogeneic mesenchymal stem cells in the brain. Neurosurgery. 2016;78(4):E602.

81. Haro-Martínez AM, Lubrini G, Madero-Jarabo R, Díez-Tejedor E, Fuentes B. Melodic intonation therapy in post-stroke nonfluent aphasia: a randomized pilot trial. Clin Rehabil. 2019;33(1):44-53.

82. Slavin D, Fabus R. A case study using a multimodal approach to melodic intonation therapy. Am J Speech Lang Pathol. 2018;27(4):1352-62.

83. Wallace SE, Donoso Brown EV, Saylor A, Lapp E. Participants' perceptions of an aphasia-friendly occupational therapy home program. Top Stroke Rehabil. 2018:1-11.

84. Northcott S, Moss B, Harrison K, Hilari K. A systematic review of the impact of stroke on social support and social networks: associated factors and patterns of change. Clin Rehabil. 2016;30(8):811-31.

85. Foley EL, Nicholas ML, Baum CM, Connor LT. Influence of environmental factors on social participation post-stroke. Behav Neurol. 2019:2019:2606039.

86. Chang HF, Power E, O'Halloran R, Foster A. Stroke communication partner training: a national survey of 122 clinicians on current practice patterns and perceived implementation barriers and facilitators. Int J Lang Commun Disord. 2018:53(6):1094-109.

87. Rose TA, Wallace SJ, Leow S. Family members' experiences and preferences for receiving aphasia information during early phases in the continuum of care. Int J Speech Lang Pathol. 2019;21(5):470-82.

88. Wang $S$, Wang $C X$, Zhang $N$, Xiang $Y T$, Yang $Y$, Shi $Y Z$, et al. The association between post-stroke depression, aphasia, and physical independence in stroke patients at 3-month follow-up. Front Psychiatry. 2018;9:374.

89. Baker C, Worrall L, Rose M, Hudson K, Ryan B, O'Byrne L. A systematic review of rehabilitation interventions to prevent and treat depression in post-stroke aphasia. Disabil Rehabil. 2018:40(16):1870-92.

90. Marshall RS, Laures-Gore J, Love K. Brief mindfulness meditation group training in aphasia: exploring attention, language and psychophysiological outcomes. Int J Lang Commun Disord. 2018;53(1):40-54.

91. Fairbairn M, Wicks E, Ait-Ouali S, Drodge O, Brooks D, Huijbregts M, et al. Facilitators of and barriers to providing access to community-based exercise programmes for adults with post-stroke aphasia from the perspective of programme representatives. Physiother Can. 2018;70(3):280-8.

92. Guina J, Guina C. Wants talk psychotherapy but cannot talk: EMDR for poststroke depression with expressive aphasia. Innov Clin Neurosci. 2018;15(1-2): $45-8$

\section{Publisher's Note}

Springer Nature remains neutral with regard to jurisdictional claims in published maps and institutional affiliations.

\section{Submit your manuscript to a SpringerOpen ${ }^{\circ}$ journal and benefit from:}

- Convenient online submission

- Rigorous peer review

- Open access: articles freely available online

- High visibility within the field

- Retaining the copyright to your article

Submit your next manuscript at $\boldsymbol{\nabla}$ springeropen.com 